\title{
Transcriptomic analysis reveals ethylene signal transduction genes involved in pistil development of pumpkin
}

\author{
Qingfei Li ${ }^{\text {Corresp., } 1,2}$, Li Zhang ${ }^{1,2}$, Feifei Pan ${ }^{1,2}$, Weili Guo ${ }^{1,2}$, Bihua Chen ${ }^{1,2}$, Helian Yang ${ }^{1,2}$, Guangyin Wang ${ }^{1,2}$, \\ Xinzheng Li ${ }^{\text {Corresp. 1, } 2}$ \\ ${ }^{1}$ College of Horticulture and Landscape[Henan Institute of Science and Technology, Xin Xiang, China \\ 2 Henan Province Engineering Research Center of Horticultural Plant Resource Utilization and Germplasm Enhancement, Xin Xiang, China \\ Corresponding Authors: Qingfei Li, Xinzheng Li \\ Email address: Iqf1988@hist.edu.cn, liuzhw@hist.edu.cn
}

Development of female flowers is an important process that directly affects the yield of Cucubits. Little information is available on the sex determination and development of female flowers in pumpkin, a typical monoecious plant. In the present study, we used aborted and normal pistils of pumpkin for RNA-Seq analysis and determined the differentially expressed genes (DEGs) to gain insights into the molecular mechanism underlying pistil development in pumpkin. A total of 3817 DEGs were identified, among which 1341 were upregulated and 2476 were downregulated. The results of transcriptome analysis were confirmed by real-time quantitative RT-PCR. KEGG enrichment analysis showed that the DEGs were significantly enriched in plant hormone signal transduction and phenylpropanoid biosynthesis pathway. Eighty-four DEGs were enriched in the plant hormone signal transduction pathway, which accounted for $12.54 \%$ of the significant DEGs, and most of them were annotated as predicted ethylene responsive or insensitive transcription factor genes. Furthermore, the expression levels of four ethylene signal transduction genes in different flower structures (female calyx, pistil, male calyx, stamen, leaf, and ovary) were investigated. The ethyleneresponsive DNA binding factor, ERDBF3, and ethylene responsive transcription factor, ERTF10, showed the highest expression in pistils and the lowest expression in stamens, and their expression levels were 78- and 162times more than that in stamens, respectively. These results suggest that plant hormone signal transduction genes, especially ethylene signal transduction genes, play an important role in the development of pistils in pumpkin. Our study provides a theoretical basis for further understanding of the mechanism of regulation of ethylene signal transduction genes in pistil development and sex determination in pumpkin. 


\title{
Transcriptomic analysis reveals ethylene signal transduction genes involved in pistil development of pumpkin
}

\author{
Qing-Fei Li ${ }^{1,2 *}$, Li Zhang1,2, Fei-Fei Pan ${ }^{1,2}$, Wei-Li Guo ${ }^{1,2}$, Bi-Hua Chen ${ }^{1,2}$, He-Lian Yang ${ }^{1,2}$, \\ Guang-Yin Wang ${ }^{1,2}$, Xin-Zheng Li $^{1,2 *}$ \\ ${ }^{1}$ College of Horticulture and Landscape, Henan Institute of Science and Technology, Xinxiang, China; \\ ${ }^{2}$ Henan Province Engineering Research Center of Horticultural Plant Resource Utilization and Germplasm \\ Enhancement, Xinxiang, China \\ * Correspondence: \\ Xin-Zheng Li, Qing-Fei Li \\ Email address: liuzhw@hist.edu.cn; lqf1988@hist.edu.cn
}

\begin{abstract}
Development of female flowers is an important process that directly affects the yield of Cucubits. Little information is available on the sex determination and development of female flowers in pumpkin, a typical monoecious plant. In the present study, we used aborted and normal pistils of pumpkin for RNA-Seq analysis and determined the differentially expressed genes (DEGs) to gain insights into the molecular mechanism underlying pistil development in pumpkin. A total of 3817 DEGs were identified, among which 1341 were upregulated and 2476 were downregulated. The results of transcriptome analysis were confirmed by real-time quantitative RT-PCR. KEGG enrichment analysis showed that the DEGs were significantly enriched in plant hormone signal transduction and phenylpropanoid biosynthesis pathway. Eighty-four DEGs were enriched in the plant hormone signal transduction pathway, which accounted for $12.54 \%$ of the significant DEGs, and most of them were annotated as predicted ethylene responsive or insensitive transcription factor genes. Furthermore, the expression levels of four ethylene signal transduction genes in different flower structures (female calyx, pistil, male calyx, stamen, leaf, and ovary) were investigated. The ethylene responsive DNA binding factor, ERDBF3, and ethylene responsive transcription factor, ERTF 10, showed the highest expression in pistils and the lowest expression in stamens, and their expression levels were 78- and 162-times more than that in stamens, respectively. These results suggest that plant hormone signal transduction genes, especially ethylene signal transduction genes, play an important role in the development of pistils in pumpkin. Our study provides a theoretical basis for further understanding of the mechanism of regulation of ethylene signal transduction genes in pistil development and sex determination in pumpkin.
\end{abstract}

Key words: Pumpkin, Pistil development, Transcriptome, Ethylene, Plant hormone signal transduction, Phenylpropanoid

\section{Introduction}


Pumpkin (Cucurbita moschata Duch.) is a typical monoecious plant with distinct male and female flowers. The number and proportion of female flowers can directly influence yields and economic benefits of Cucurbitaceae crops. Pistil is the main characteristic structure of female flowers. It is well established that ethylene promotes pistil and female flower development. Application of ethylene, or inhibition of ethylene action, increases or decreases the number of pistil-bearing buds (Papadopoulou et al., 2005). Ethylene promotes carpel development and arrests stamen development in female flower (Chen et al., 2016). Treatment with exogenous ethylene or ethylene releasing reagents can increase the numbers of female and bisexual flowers in monoecious and andromonoecious lines, respectively (Iwahori et al., 1969;Malepszy and Niemirowicz-Szczytt, 1991; Manzano et al., 2011). Auxin response factors (ARFs), an important component in auxin signalling pathway, especially the $A R F 13$ and $A R F 17$ genes are essential for pistil development in Japanese apricot (Song et al., 2015). The development of female flowers is inseparable from sex differentiation in Cucurbitaceae crops.

A number of studies have found that the floral development and sex expression in Cucurbitaceae crops can be affected by multiple phytohormones, including ethylene, auxin, cytokinin, gibberellin, abscisic acid, brassinosteroid, jasmonic acid, and salicylic acid (Rudich and Halevy, 1974;Trebitsh et al., 1987;Yamasaki et al., 2003;Menéndez et al., 2009;Pimenta Lange and Lange, 2016;Mao et al., 2017;Zhang et al., 2017). Ethylene might be the major hormone in sex determination. In cucumber, sex differentiation is mainly determined by the $F$ (CsACS1G), $M$ (CsACS2), and $A$ (CsACS11) genes. The $F, M$, and $A$ genes encode 1aminocyclopropane-1-carboxylate (ACC) synthase (ACS), which is a key rate-limiting enzyme in ethylene biosynthetic pathway (Pierce and Wehner, 1990;Pan et al., 2018). Among these, the $F$ gene promotes the development of female flowers (Mibus and Tatlioglu, 2004;Knopf and Trebitsh, 2006), and the $M$ gene inhibits the development of stamens (Yamasaki et al., 2001; Saito et al., 2007). The $\operatorname{Cs} A C S 11(A)$ is an androecious gene, and mutants with loss of CsACSI 1 function were found to be androecious, with no female flowers (Boualem et al., 2015). The ACC oxidase gene $(A C O)$, another key gene in ethylene biosynthesis, is also essential for the development of female flowers. A transcription factor gene, CsWIP1, could directly bind the promoter of $\mathrm{CsACO}_{2}$ to repress its expression (Chen et al., 2016). In melon, sex determination is governed by the genes, andromonoecious (a) and gynoecious (g). CmACS-7 is the andromonoecious gene (Boualem et al., 2008). CmACS1 1 controls the development of female flowers in melon and its function is not exactly the same as of CsACS11.CmWIP1, the ortholog of $C s W I P 1$, can negatively regulate the femaleness and gynoecious plants are obtained when there is a loss of function of CmWIP1. The expression patterns of CmWIP1 and CmACSI1 are opposite in melon. CmACS11 can repress the expression of CmWIP1 at an upstream step in the sex determination pathway to control flower development. Ethylene could inhibite stamen development through CmACS-7 and CmACS11 (Boualem et al., 2015). Thus, the sex differentiation and floral development in melon could be a result of the interaction among CmACS11, CmWIP1, and CmACS-7. 
Besides the genes involved in ethylene synthesis, those involved in ethylene signal transduction have also been implicated in the development of female flowers in cucumber. Previous studies have revealed that there existed organ-specific DNA damage in primordial anther of female flowers and that the DNA damage was induced via the ethylene signaling pathway. The ethylene-receptor gene, CsETR1, located in the pistil primordia has also been involved in the arrest of stamen development through induction of DNA damage in female flowers (Hao et al., 2003; Yamasaki et al., 2003;Duan et al., 2008;Wang et al., 2010). It can be bound and activated by CSAP3 in vitro and in vivo (Sun et al., 2016). Moreover, the expression of $C_{S^{-}} E T R 2$ and $C_{S^{-}} E R S$ was reported to be regulated by ethylene, because their mRNAs were significantly elevated by the application of ethrel and their levels were lowered by the application of an ethylene inhibitor, aminoethoxyvinyl glycine (Yamasaki et al., 2000). Thus, not only the genes involved in ethylene synthesis, those involved in ethylene-mediated signal transduction contribute to the development of female flowers. However, the evidence for how the ethylene signal transduction genes regulate the development of female flowers is still weak.

Besides ethylene, other phytohormones are also involved in the development of female and male flowers. Gibberellins (GA) can promote the male tendency. Its production in andromonoecious cucumber is higher than that in monoecious and gynoecious cucumber (Hemphill et al., 1972). A recent report indicated that GA could regulate sex expression via ethylene-dependent and ethylene-independent pathways (Zhang et al., 2017). The application of indole-3-acetic acid (IAA), except for the Beta -Alfa type, could enhance ethylene and ACC production (Trebitsh et al., 1987). Cytokinins are possibly involved in determining the morphological differences between sex types. The endogenous levels of the cytokinins were found to be higher in female gametophytes than in male gametophytes (Menéndez et al., 2009). Abscisic acid could promote the female tendency of gynoecious plants. It participates in the sex regulation by inhibiting the GA activity of cucumber (Rudich and Halevy, 1974). Jasmonic acid signaling also plays an important role in flower development in plants, especially in stamen sterility, sex determination, female flower development, and seed maturation (Yuan and Zhang, 2015; Mao et al., 2017).

As evident from the above studies, floral development and sex differentiation is a result of the interaction of various plant hormones and ethylene apparently plays a key role in these processes. However, little is known about how the plant hormone signal transduction genes are involved in female and male flower development. Herein, to explore the key genes involved in the development of pistils, the aborted and normal pistils of pumpkin were used for RNA-Seq analysis. The results showed that plant hormone signal transduction genes, especially ethylene related genes, play important roles in the development of pistils in pumpkin. Fourteen DEGs, which were annotated as plant hormone signal transduction genes, were chosen for qRT-PCR verification. Furthermore, four ethylene signal transduction genes were appealing candidates for investigation of their expression in different flower structures. Our results provide a foundation for dissecting the molecular mechanism of regulation of pistil formation in pumpkin by the 
117

118

119

120

121

122

123

124

125

126

127

128

129

130

131

132

133

134

135

136

137

138

139

140

141

142

143

144

145

146

147

148

candidate genes, identified herein.

\section{Materials and Methods}

\section{Plant materials}

The aborted plants were from the same inbred line as the normal plants and exhibited similar plant characters except for the female development. The seeds of pumpkin were sterilized with hot water $\left(55^{\circ} \mathrm{C}\right)$ for approximately $15 \mathrm{~min}$, and then soaked in water for $4 \mathrm{~h}$. The seeds were germinated on a wet filter paper in a Petri dish at $28^{\circ} \mathrm{C}$ in the dark. When at least $80 \%$ of the seeds had germinated, they were transferred into a substrate mixture of peat, vermiculite, and perlite $(3: 1: 0.5, \mathrm{v} / \mathrm{v})$ and grown in an artificial climate room under conditions of 14-h light/10-h dark and day/night temperatures of $25^{\circ} \mathrm{C} / 18^{\circ} \mathrm{C}$. When the seedlings had developed three true leaves, they were transplanted to a field and grown under the natural environment. At flower development period, the aborted female flowers exhibited different from those of the normal plants. When the length of corolla of flower bud was less than $5 \mathrm{~mm}$, there was no difference between the aborted female flower and the normal one. As the buds grow bigger, in aborted pistils the incomplete stigma can be seen when the corolla is peeled off, and ovary looks thinner than in normal pistils, which is as shown in Fig. 1A. Thus, the aborted and normal pistils, which were come from the female flower buds with $5 \mathrm{~mm}$ length of corolla and located in the shoot apices, were used for RNA-Seq analysis and for the validation of gene expression. Moreover, leaf, female calyx, pistil, ovary, male calyx, and stamen from the male and female flowers of the same plants, were used to detect the expression of the candidate genes in different floral structures. All the samples were immediately frozen in liquid nitrogen and stored at $-80{ }^{\circ} \mathrm{C}$ for further experiments.

\section{RNA isolation}

Total RNA was extracted using the Trizol ${ }^{\mathrm{TM}}$ reagent (Invitrogen, Carlsbad, CA, USA), as described by the manufacturer. DNase I was then used to remove DNA contamination in the RNA preparations. The purity and integrity of RNA samples were evaluated by electrophoresis on 1\% RNase-free agarose gels, spectrophotometry (A260/A280 and A260/A230) on NanoDrop DU8000, and analysis using the Agilent 2100 Bioanalyzer system (Agilent, USA).

\section{Construction and sequencing of cDNA libraries}

The NEBNext Ultra Directional RNA Library Prep Kit for Illumina (NEB, Ispawich, USA) was used for mRNA fragmentation. In brief, poly (A) mRNA was extracted from total RNA using oligo-dT magnetic beads, and further fragmented. First strand cDNA was synthesized using 
random hexamer primers and reverse transcriptase. Second strand cDNA was synthesized using dNTPs, DNA polymerase I, RNase H, and the first strand. To select the fragments that were preferentially 150-200 bp in length, the cDNA fragments were purified with an AMPure XP system (Beckman Coulter, USA). The high-quality libraries were then sequenced on the Illumina HiSeq 4000 platform by Sagene Co. (Guangzhou, China). The libraries of two biological replicates of aborted and normal pistils were prepared independently. The raw transcriptome data were deposited in the National Center for Biotechnology Information Sequence Read Archive under BioProject number PRJNA554766.

\section{Bioinformatics analysis of differentially expressed genes (DEGs) data}

The raw reads were pre-processed by removing the adaptor sequences, low-quality reads (more than $50 \%$ bases with $\mathrm{SQ} \leq 20$ in one sequence), and reads with more than $5 \% \mathrm{~N}$ bases (bases unknown). The clean reads were mapped to the Cucurbita moschata genome (http://cucurbitgenomics.org/organism/9) using TopHat2 v2.1.1 (Trapnell et al., 2012;Kim et al., 2013; Sun et al., 2017), allowing up to two mismatch. Functional annotation was performed by searching against the non redundant (NR), Swiss-Prot and clusters of orthologous groups for eukaryotic complete genomes (KOG) databases using BLAST with an E-value of $1 \mathrm{e}^{-5}$. The R package edgeR was used to identify the DEGs using raw read counts as input data (Robinson et al., 2010). P-values were adjusted using Benjamini and Hochberg's method to control the false discovery rate (FDR) (Benjamini and Hochberg, 1995). The $\mid \log 2$ (fold change) $\mid>1$ and adjusted P-value $<0.05$ for multiple tests using the Benjamini method were used as significance cut-offs for the expression differences.

\section{Functional enrichment analysis for DEGs}

Gene functional enrichment analysis of DEGs was implemented using the GOSeq R package (Young et al., 2010). GO terms included cellular component, molecular function, and biological process. After the hypergeometric test, Bonferroni correction was employed for P-value correction with a cut-off of 0.05 . The GO terms satisfying the condition were considered significantly enriched by DEGs. Furthermore, the Kyoto Encyclopedia of Genes and Genomes (KEGG) enrichment analysis was performed. KEGG is the main public database of pathways. FDR control method was used to identify the threshold of the P-value in multiple tests (Benjamini and Hochberg, 1995). Pathways with their Benjamini and Hochberg adjusted Pvalues $\leq 0.05$ were defined as significantly enriched by DEGs (Mao et al., 2005;Kanehisa et al., 2014). Using significant enrichment of pathways, the major biochemical metabolic pathways and signal transduction pathways involving DEGs can be determined.

\section{Quantitative real-time RT-PCR}

RNA extraction and detection were done as described above. cDNA synthesis was conducted using the PrimeScript ${ }^{\mathrm{TM}}$ RT Master Mix (Perfect Real Time) Reagent Kit (Takara, Dalian, China), according to the manufacturer's instructions. The gene primers used in the quantitative real-time 
186

187

188

189

190

191

192

193

194

195

196

197

198

199

200

201

202

203

204

205

206

207

208

209

210

211

212

213

214

215

216

217

218

219

220

221

222

RT-PCR (qRT-PCR) experiment are listed in Supplemental Table 1. ACTIN was used as an internal control. The qRT-PCR system was consisted of $10 \mu \mathrm{L}$ SYBR Premix Ex Taq ${ }^{\mathrm{TM}}$ II, 0.8 $\mu \mathrm{L}$ each primer $(10 \mu \mathrm{M}), 2 \mu \mathrm{L}$ diluted cDNA (150 ng), and $6.4 \mu \mathrm{L}$ nuclease-free water. The qRTPCR reactions were performed in three biological replicates. The qRT-PCR was carried out using a Bio-Rad IQ5 instrument (Foster City, CA, USA), with the following conditions: 1 cycle of $95^{\circ} \mathrm{C}$ for $40 \mathrm{~s}, 40$ cycles of $95^{\circ} \mathrm{C}$ for $5 \mathrm{~s}$ and $61^{\circ} \mathrm{C}$ for $30 \mathrm{~s}$. The relative expression levels were calculated using the $2^{-\Delta \Delta \mathrm{Ct}}$ method (Livak and Schmittgen, 2001). To verify the RNA-Seq data, the correlation analysis and the Pearson correlation coefficient between log 2 (fold change) of RNA-Seq and qRT-PCR was calculated using the IBM SPSS statistics 22 software.

\section{Results}

\section{Summary of the sequencing data}

After filtering, 5.62, 6.60, 5.86, and 5.72 million high quality clean reads for aborted (A2, A4) and normal $(\mathrm{N} 2, \mathrm{~N} 4)$ pistils were obtained. Among these high quality clean reads, the percentages of Q20 were $97.46 \%, 97.58 \%, 96.61 \%$, and $97.45 \%$, respectively, and the percentage of GC were $45.55 \%, 45.49 \%, 45.77 \%$, and $45.81 \%$, respectively. The total pair reads, mapped pair reads (ratio) of these libraries according to the Cucurbita moschata genome, known gene number, and number of novel genes were as shown in Table 1. Finally, 3817 DEGs were identified, including 1341 up-regulated and 2476 down-regulated genes, in the aborted pistils compared with the normal pistils (Fig. 1B, C, Supplemental Table 2).

\section{GO enrichment analysis}

The DEGs were enriched in cellular component, molecular function, and biological process groups. A total of 749 DEGs were categorized into biological process category, which mainly included metabolic process (213 up-regulated, 345 down-regulated), cellular process (179 upregulated, 307 down-regulated), and single-organism process (151 up-regulated, 235 downregulated) (Fig. 2). Among these, the most significantly enriched GO terms included singleorganism metabolic process $(256, P=9.21 \mathrm{E}-12)$, hydrogen peroxide metabolic process $(16, P=$ $7.80 \mathrm{E}-07)$, phenylpropanoid metabolic process $(10, P=4.34 \mathrm{E}-04)$, and carbohydrate metabolic process $(49, P=4.41 \mathrm{E}-04) .580$ DEGs were divided into the cellular component category, and they mainly enriched in membrane (140 up-regulated, 260 down-regulated), membrane part (111 up-regulated, 188 down-regulated), and cell (94 up-regulated, 146 down-regulated) terms (Fig. $2)$. The most significantly enriched GO terms in the cellular component category included extracellular region $(19, P=5.01 \mathrm{E}-05)$, membrane (400, $P=3.94 \mathrm{E}-04)$, photosystem II $(6, P=$ 5.37E-03), and external encapsulating structure $(23, P=9.34 \mathrm{E}-03)$. A total of 762 DEGs were divided into molecular function, which mainly enriched in the catalytic activity (205 upregulated, 362 down-regulated), binding (178 up-regulated, 235 down-regulated), and transporter activity (26 up-regulated, 35 down-regulated). The most significantly enriched GO terms in the molecular function category included oxidoreductase activity $(152, P=1.47 \mathrm{E}-17)$, tetrapyrrole 
binding $(56, P=1.69 \mathrm{E}-12)$, ion binding $(186, P=6.80 \mathrm{E}-05)$, and cation binding $(167, P=1.96 \mathrm{E}-$ 04) (Fig. 2). On the whole, in cellular component, molecular function, and biological process groups, the down-regulated genes were all more than up-regulated ones.

\section{Plant hormone signal transduction and phenylpropanoid biosynthesis involved in pistil development in pumpkin}

The KEGG pathway enrichment analysis indicated that the DEGs were significantly enriched in the following pathways: phenylpropanoid biosynthesis, plant hormone signal transduction, phenylalanine metabolism, tryptophan metabolism, linoleic acid metabolism (Fig. 3). It showed that 84 DEGs were enriched in the plant hormone signal transduction pathway (Supplemental Table 3), which accounted for $12.54 \%$ of all the pathway annotated DEGs. The plant hormones involved in this pathway included ethylene, auxin, cytokinin, gibberellin, abscisic acid, brassinosteroid, jasmonic acid, and salicylic acid. Most of DEGs enriched in the plant hormone signal transduction pathway were annotated as predicated ethylene-responsive transcription factor, ethylene insensitive transcription factor, and ethylene receptor genes in NCBI blast and Cucurbita moschata genome (Supplemental Table 4, Supplemental Fig. 1).

In addition, we found that 68 DEGs were enriched in phenylpropanoid biosynthesis (Supplemental Table 5), which accounted for $10.15 \%$ of all the pathway annotated DEGs. Among these, seven DEGs were defined as phenylalanine ammonia-lyase genes $(P A L)$, which coded for the key first rate-limiting enzyme in the phenylpropanoid pathway. It is worth noting that six $P A L$ DEGs were downregulated in the aborted pistils (Table 2). The results indicated that the phenylpropanoid pathway, especially $P A L$, might be implicated in pistil development in pumpkin.

\section{Verification of RNA-seq data by qRT-PCR analyses}

The RNA-Seq results were validated by qRT-PCR assays. Fourteen annotated DEGs were chosen for qRT-PCR analyses. Twelve of these genes were downregulated and two were upregulated. It included auxin related genes, $A U X$-IAA (CmoCh04G015610, auxin-responsive protein IAA gene) and $A R F$ (CmoCh16G005850, auxin response factor), gibberellins-related gene, $T F$ (CmoCh05G010630, PIF4: phytochrome-interacting factor 4), ethylene related genes, $A C O$ (CmoCh02G000640, 1-aminocyclopropane-1-carboxylate oxidation), ETR (CmoCh08G004320, ethylene receptor), ERDBF3 (CmoCh17G005350, ethylene response DNA binding factor), ERTF10 (CmoCh11G005450, ethylene responsive transcription factor gene), and AP2 (CmoCh01G000430, ethylene responsive transcription factor gene), brassinosteroid biosynthesis genes, TCH4 (CmoCh11G004020, xyloglucan:xyloglucosyl transferase) and CYCD3 (CmoCh01G018690, cyclin D3), jasmonic acid pathway gene, JAR1 (CmoCh06G010560, jasmonic acid-amino synthetase), salicylic acid pathway gene, NPR1 (CmoCh03G001860, regulatory protein), and transcription factor, TGA (CmoCh13G003300), and phenylpropanoid biosynthesis gene, $P A L$ (CmoCh07G009540, phenylalanine ammonia- 
260

261

262

263

264

265

266

267

268

269

270

271

272

273

274

275

276

277

278

279

280

281

282

283

284

285

286

287

288

289

290

291

292

293

294

295

lyase). As shown in Fig. 4, all the detected genes showed similar expression trends in qRT-PCR analyses as in RNA-Seq data, with a relative coefficient of $R^{2}=0.7887$ (Fig. 4A, B). The Pearson correlation analysis indicated that the RNA-Seq and qRT-PCR were strongly correlated $(R=$ $0.888, P=0.00002)$.

Expression levels of ethylene candidate genes in different flower structures

Ethylene signal transduction genes (ERDBF3, ERTF10, AP2, and $E T R)$ were appealing candidates for investigating their gene expression in different flower structures, including pistil, female calyx, ovary, stamen, male calyx, and leaf. The results showed that the expression level of ethylene response DNA binding factor, $E R D B F 3$, was highest in pistil, followed by that in male calyx, leaf, female calyx, ovary, and stamen (Fig. 5), and the expression level in pistil was 78-times more than that in stamen. The expression level of ERTF10 was also highest in pistils, followed by that in leaf, male calyx, female calyx, ovary, and stamen (Fig. 5), and the expression level in pistil was 162-times more than that in stamen. ERTF10 and ERDBF3 showed the highest expression in pistil and the lowest in stamen. $A P 2$ has been reported in other crops to be important for flower and seed development (Jofuku et al., 1994; Zhang et al., 2018). In this study, the expression level of $A P 2$ was found to be higher in the reproductive organ stamen, male calyx, female calyx, pistil, and ovary compared to that in leaf (Fig. 5). This indicates that, in pumpkin,

$A P 2$ may also play an important role in flower development. The expression level of the ethylene receptor, ETR, was highest in stamen, followed by that in leaf, ovary, female calyx, pistil, and male calyx (Fig. 5). From the results, we can see that the four ethylene signal transduction genes are important for the development of female and male flowers, and ERTF10 and ERDBF3 may especially be more important in the pistils of female flowers than that in the stamens of male flowers.

\section{Discussion}

The number and proportion of female flowers are directly related to the yield of Cucurbitaceae crops. In previous studies, researchers have focused on sex differentiation to explore the key genes and mechanisms regulating the development of female and male flowers. Ethylene is known to promote the development of female flowers, which was mainly manifested in sexual expression, the earliness and the larger number of female flowers per plant. Moreover, the development of female flowers requires much more ethylene than the development of male flowers (Pierce and Wehner, 1990;Malepszy and Niemirowicz-Szczytt, 1991;Manzano et al., 2011; Manzano et al., 2014;Pan et al., 2018).

In this study, the key genes involved in pistil development were studied directly using aborted pistil materials. The results of KEGG pathway analysis showed that the DEGs were enriched in plant hormone signal transduction pathway, and most of them were annotated as predicated ethylene-responsive transcription factor, ethylene insensitive transcription factor, and ethylene 
296

297

298

299

300

301

302

303

304

305

306

307

308

309

310

311

312

313

314

315

316

317

318

319

320

321

322

323

324

325

326

327

328

329

330

331

332

receptor genes (Supplemental Table 4). This result echo a previous study which have suggested that ethylene synthesis and signal transduction play important roles in sex expression of pumpkin (Cucurbita maxima) (Wang et al., 2019). Furthermore, we found that the ethylene response DNA binding factor, $E R D B F 3$, and the ethylene responsive transcription factor, ERTF10, showed the highest expression in pistils and the lowest expression in stamens (Fig. 5). These results indicate that ethylene signal transduction genes, ERTF10 and ERDBF3, may be more important in the pistils of female flowers than in other floral structures. In addition, previous studies have also shown that the ethylene receptor, CsETR1, and the ethylene-responsive transcription factor, $C s A P 3$, are involved in the development of female flowers (Hao et al., 2003; Yamasaki et al., 2003; Wang et al., 2010;Sun et al., 2016). The results presented in Fig. 5 show that AP2 and ETR were expressed in all the structures of flowers that were assessed, and although their expression level in the stamens was higher than that in the pistils, there is not enough evidence to show as to how important they are for the development of female and male flowers. Except for the ethylene signal transduction, which might play a major role in the development of female flowers, the results of KEGG analysis also indicated that other plant hormone signal transduction pathways, including auxin, cytokinin, gibberellin, abscisic acid, brassinosteroid, jasmonic acid, salicylic acid signaling pathways, are involved in flower development. There is a possibility for the existence of interactions between various plant hormones through their signal transduction pathways. For example, ethylene synthesis can be induced by auxin, gibberellin, and jasmonic acid (Rudich and Halevy, 1974; Trebitsh et al., 1987;Zhang et al., 2017;Schubert et al., 2019).

Phenylpropanoids comprise many aromatic metabolites, including the cell wall structural component, lignin, and many small phenolic molecules, such as coumarins, stilbenes, flavonoids, anthocyanins, and condensed tannins (Vogt, 2010; Fraser and Chapple, 2011;Zhang et al., 2013). Phenylalanine ammonia-lyase catalyzes the first rate-limiting step in the phenylpropanoid pathway, which controls the carbon flux to aromatic compounds, and to lignin. The results of this study indicate that six PAL DEGs are downregulated in aborted pistils (Table 2). Previous study have indicated that transgenic tobacco plants carrying antisense and sense pal cDNAs resulted in partial male sterility, with the reduction of pollen fertility (Matsuda et al., 1996). At present, there has been no systematic research on the mechanisms through which phenylpropanoids take part in the development of female flowers.

In addition, the DEGs in the present study were obtained from RNA-Seq analysis of the aborted and normal pistils, which were come from the flower buds with $5 \mathrm{~mm}$ length of corolla. More genes related to pistil development were mined. But, when the aborted female flower buds grew bigger, they exhibited incomplete stigma and thinner ovary for the absence of ovules, and which is the primary cause of pistil abortion is unclear. Further gene function analysis is needed to illuminate the mechanism of pistil abortion.

\section{Conclusions}


In the present study, DEGs in aborted and normal pistils of pumpkin were identified. KEGG enrichment analysis showed that the DEGs were significantly enriched in plant hormone signal transduction, which accounted for $12.54 \%$ of the significant DEGs. Most of them were annotated as predicated ethylene signal transduction genes. The expression analysis of ERDBF3 and ERTF 10 in different flower structures showed the highest expression in pistils and the lowest expression in stamens. These results suggest that plant hormone signal transduction genes, especially ethylene signal transduction genes, play an important role in the development of pistils in pumpkin.

\section{References}

Benjamini Y, Hochberg Y. 1995. Controlling the false discovery rate: a practical and powerful approach to multiple testing. Journal of the Royal statistical society: series $B$ (Methodological) 57:289-300. DOI: 10.1111/j.2517-6161.1995.tb02031.x.

Boualem A, Fergany M, Fernandez R, Troadec C, Martin A, Morin H, Sari MA, Collin F, Flowers JM, Pitrat M, Purugganan MD, Dogimont C, Bendahmane A. 2008. A conserved mutation in an ethylene biosynthesis enzyme leads to andromonoecy in melons. Science 321:836-838. DOI: 10.1126/science.1159023.

Boualem A, Troadec C, Camps C, Lemhemdi A, Morin H, Sari MA, Fraenkel-Zagouri R, Kovalski I, Dogimont C, Perl-Treves R, Bendahmane A. 2015. A cucurbit androecy gene reveals how unisexual flowers develop and dioecy emerges. Science 350:688-691. DOI: 10.1126/science.aac8370.

Chen H, SunJ, Li S, Cui Q, Zhang H, Xin F, Wang H, Lin T, Gao D, Wang S, Li X, Wang D, Zhang Z, Xu Z, Huang S. 2016. An ACC oxidase gene essential for cucumber carpel development. Molecular Plant 9:1315-1327. DOI: 10.1016/j.molp.2016.06.018.

Duan QH, Wang DH, Xu ZH, Bai SN. 2008. Stamen development in Arabidopsis is arrested by organ-specific overexpression of a cucumber ethylene synthesis gene CsACO2. Planta 228:537-543. DOI: 10.1007/s00425-008-0756-7.

Fraser CM, Chapple C. 2011. The phenylpropanoid pathway in arabidopsis. The Arabidopsis Book/American Society of Plant Biologists 9:e0152. DOI: 10.1199/tab.0152.

Hao YJ, Wang DH, Peng YB, Bai SL, Xu LY, Li YQ, Xu ZH, Bai SN. 2003. DNA damage in the early primordial anther is closely correlated with stamen arrest in the female flower of cucumber (Cucumis sativus L.). Planta 217:888-895. DOI: 10.1007/s00425-003-1064-x.

Hemphill DD, Baker LR, Sell HM. 1972. Different sex phenotypes of Cucumis sativus L. and C. melo L. and their endogenous gibberellin activity. Euphytica 21:285-291. DOI: 10.1007/BF00036769.

Iwahori S, Lyons JM, Sims WL. 1969. Induced femaleness in cucumber by 2chloroethanephosphonic acid. Nature 222:271-272. DOI: 10.1038/222271a0. 
Jofuku KD, den Boer BG, Van Montagu M, Okamuro JK. 1994. Control of Arabidopsis flower and seed development by the homeotic gene APETALA2. The Plant Cell 6:1211-1225. DOI: 10.1105/tpc.6.9.1211.

Kanehisa M, Goto S, Sato Y, Kawashima M, Furumichi M, Tanabe M. 2014. Data, information, knowledge and principle: back to metabolism in KEGG. Nucleic Acids Research 42: 199205. DOI: 10.1093/nar/gkt1076.

Kim D, Pertea G, Trapnell C, Pimentel H, Kelley R, Salzberg SL. 2013. Tophat2: accurate alignment of transcriptomes in the presence of insertions, deletions and gene fusions. Genome biology 14:R36. DOI: 10.1186/gb-2013-14-4-r36.

Knopf RR, Trebitsh T. 2006. The female-specific CS-ACS1g gene of cucumber. A case of gene duplication and recombination between the non-sex-specific 1-aminocyclopropane-1carboxylate synthase gene and a branched-chain amino acid transaminase gene. Plant \&Cell Physiology 47, 1217-1228. DOI: 10.1093/pcp/pcj092.

Livak KJ, SchmittgenTD. 2001. Analysis of relative gene expression data using real-time quantitative PCR and the 2(-Delta Delta C(T)) method. Methods 25:402-408. DOI: 10.1006/meth.2001.1262.

Malepszy S, Niemirowicz-Szczytt K. 1991. Sex determination in cucumber (Cucumis sativus) as a model system for molecular biology. Plant Science 80:39-47. DOI: 10.1016/01689452(91)90271-9.

Manzano S, Martínez C, García JM, Megías Z, Jamilena M. 2014. Involvement of ethylene in sex expression and female flower development in watermelon (Citrullus lanatus). Plant Physiology and Biochemistry 85:96-104. DOI: 10.1016/j.plaphy.2014.11.004.

Manzano S, Martínez C, Megías Z, Gómez P, Garrido D, Jamilena M. 2011. The role of ethylene and brassinosteroids in the control of sex expression and flower development in Cucurbita pepo. Journal of Plant Growth Regulation 65: 213-221. DOI: 10.1007/s10725-011-9589-7.

Mao X, Cai T, Olyarchuk J, Wei L. 2005. Automated genome annotation and pathway identification using the KEGG Orthology $(\mathrm{KO})$ as a controlled vocabulary. Bioinformatics 21:3787-3793. DOI: 10.1093/bioinformatics/bti430.

Mao Y, Liu W, Chen X, Xu Y, Lu W, Hou J, Ni J, Wang Y, Wu L. 2017. Flower development and sex determination between male and female flowers in Vernicia fordii. Frontiers in Plant Science 8:1291. DOI: 10.3389/fpls.2017.01291.

Matsuda N, Tsuchiya T, Kishitani S, Tanaka Y, Toriyama K. 1996. Partial male sterility in transgenic tobacco carrying antisense and sense $P A L$ cDNA under the control of a tapetumspecific promoter. Plant and Cell Physiology 37:215-222. DOI: 10.1093/oxfordjournals.pcp.a028934.

Menéndez V, Revilla MA, Fal MA, Fernández H. 2009. The effect of cytokinins on growth and sexual organ development in the gametophyte of Blechnum spicant L. Plant Cell, Tissue and Organ Culture 96:245-250. DOI: 10.1007/s11240-008-9481-y. 
408

409

410

411

412

413

414

415

416

417

418

419

420

421

422

423

424

425

426

427

428

429

430

431

432

433

434

435

436

437

438

439

440

441

442

443

444

445

446

Mibus H, Tatlioglu T. 2004. Molecular characterization and isolation of the F/f gene for femaleness in cucumber (Cucumis sativus L.). Theoretical And Applied Genetics 109:16691676. DOI: $10.1007 / \mathrm{s} 00122-004-1793-7$.

Pan J, Wang G, Wen H, Du H, Lian H, He H, Pan J, Cai R. 2018. Differential gene expression caused by the $F$ and $M$ loci provides insight into ethylene-mediated female flower differentiation in cucumber. Frontiers in Plant Science 9:1091. DOI: 10.3389/fpls.2018.01091.

Papadopoulou E, Little HA, Hammar SA, Grumet R. 2005. Effect of modified endogenous ethylene production on sex expression, bisexual flower development and fruit production in melon (Cucumis melo L.). Sexual Plant Reproduction 18: 131-142. DOI: 10.1007/s00497005-0006-0.

Pierce L, Wehner T. 1990. Review of genes and linkage groups in cucumber. Horticultural Science 25:605-615. DOI: 10.21273/HORTSCI.25.6.605.

Pimenta Lange MJ, Lange T. 2016. Ovary-derived precursor gibberellin A9 is essential for female flower development in cucumber. Development 143:4425-4429. DOI: 10.1242/dev.135947.

Robinson MD, McCarthy DJ, Smyth GK. 2010. EdgeR: a bioconductor package for differential expression analysis of digital gene expression data. Bioinformatics 26: 139-140. DOI: 10.1093/bioinformatics/btp616.

Rudich J, Halevy AH. 1974. Involvement of abscisic acid in the regulation of sex expression in the cucumber. Plant \&Cell Physiology 15:635-642. DOI: 10.1093/oxfordjournals.pcp.a075048.

Saito S, Fujii N, Miyazawa Y, Yamasaki S, Matsuura S, Mizusawa H, FujitaY, Takahashi H. 2007. Correlation between development of female flower buds and expression of the CSACS2 gene in cucumber plants. Journal of Experimental Botany 58:2897-2907. DOI: $10.1093 / \mathrm{jxb} / \mathrm{erm} 141$.

Schubert R, Grunewald S, von Sivers L, Hause B. 2019. Effects of jasmonate on ethylene function during the development of tomato stamens. Plants (Basel) 8. DOI: $10.3390 /$ plants 8080277 .

Song J, Gao ZH, Huo XM, Sun HL, Xu YS, Shi T, Ni ZJ. 2015. Genome-wide identification of the auxin response factor $(A R F)$ gene family and expression analysis of its role associated with pistil development in Japanese apricot (Prunus mume Sieb. et Zucc). Acta Physiologiae Plantarum 37: 145. DOI: 10.1007/s11738-015-1882-Z.

Sun H, Wu S, Zhang G, Jiao C, Guo S, Ren Y, Zhang J, Zhang H, Gong G, Jia Z, Zhang F, Tian J, Lucas WJ, Doyle JJ, Li H, Fei Z, Xu Y. 2017. Karyotype stability and unbiased fractionation in the paleo-allotetraploid Cucurbita genomes. Molecular Plant 10:1293-1306. DOI: 10.1016/j.molp.2017.09.003.

Sun JJ, Li F, Wang DH, Liu XF, Li X, Liu N, Gu HT, Zou C, Luo JC, He CX, Huang SW, Zhang XL, Xu ZH, Bai SN. 2016. CsAP3: a cucumber homolog to Arabidopsis 
APETALA3 with novel characteristics. Frontiers in Plant Science 7:1181. DOI: 10.3389/fpls.2016.01181.

Trapnell C, Roberts A, Goff L, Pertea G, Kim D, Kelley DR, Pimentel H, Salzberg SL, Rinn JL, Pachter L. 2012. Differential gene and transcript expression analysis of RNA-seq experiments with TopHat and Cufflinks. Nature Protocols 7:562-578. DOI: 10.1038/nprot.2012.016.

Trebitsh T, Rudich J, Riov J. 1987. Auxin, biosynthesis of ethylene and sex expression in cucumber (Cucumis sativus). Plant Growth Regulation 5:105-113. DOI: $10.1007 / \mathrm{BF} 00024738$.

Vogt T. 2010. Phenylpropanoid biosynthesis. Molecular Plant 3:2-20. DOI: 10.1093/mp/ssp106. Wang DH, Li F, Duan QH, Han T, Xu ZH, Bai SN. 2010. Ethylene perception is involved in female cucumber flower development. Plant Journal 61:862-872. DOI: 10.1111/j.1365313X.2009.04114.X.

Wang YL, Yan CD, Zou BX, Wang CJ, Xu WL, Cui CS, Qu SP. 2019 Morphological, transcriptomic and hormonal characterization of trimonoecious and subandroecious pumpkin (Cucurbita maxima) suggests important roles of ethylene in sex expression. International Journal of Molecular Science 20:3185. DOI: 10.3390/ijms20133185.

Yamasaki S, Fujii N, Takahashi H. 2000. The ethylene-regulated expression of CS-ETR2 and $C S-E R S$ genes in cucumber plants and their possible involvement with sex expression in flowers. Plant and Cell Physiology 41:608-616. DOI: 10.1093/pcp/41.5.608.

Yamasaki S, Fujii N, Takahashi H. 2003. Characterization of ethylene effects on sex determination in cucumber plants. Sexual Plant Reproduction 16:103-111. DOI: 10.1007/s00497-003-0183-7.

Yamasaki S, Fujii N, Matsuura S, Mizusawa H, Takahashi H. 2001. The M locus and ethylenecontrolled sex determination in andromonoecious cucumber plants. Plant and Cell Physiology 42:608-619. DOI: 10.1093/pcp/pce076.

Young MD, Wakefield MJ, Smyth GK, Oshlack A. 2010. Gene ontology analysis for RNA-seq: accounting for selection bias. Genome Biology 11, R14. DOI: 10.1186/gb-2010-11-2-r14.

Yuan Z, Zhang D. 2015. Roles of jasmonate signalling in plant inflorescence and flower development. Current Opinion in Plant Biology 27:44-51. DOI: 10.1016/j.pbi.2015.05.024.

Zhang X, Gou M, Liu CJ. 2013. Arabidopsis Kelch repeat F-box proteins regulate phenylpropanoid biosynthesis via controlling the turnover of phenylalanine ammonia-lyase. Plant Cell 25:4994-5010. DOI: 10.1105/tpc.113.119644.

Zhang Y, Huang S, Wang X, Liu J, Guo X, Mu J, Tian J, Wang X. 2018. Defective APETALA2 genes lead to sepal modification in brassica crops. Frontiers in Plant Science 9:367. DOI: 10.3389/fpls.2018.00367.

Zhang Y, Zhao G, Li Y, Mo N, Zhang J, Liang Y. 2017. Transcriptomic analysis implies that GA regulates sex expression via ethylene-dependent and ethylene-independent pathways in cucumber (Cucumis sativus L.). Frontiers in Plant Science 8:10. DOI: 10.3389/fpls.2017.00010. 


\section{Table $\mathbf{1}$ (on next page)}

Statistical results of reads mapped to reference genome and expressed genes in different libraries.

Statistical results of reads mapped to reference genome after rRNA filtering and expressed genes in different libraries. 


\begin{tabular}{ccccc}
\hline Sample & $\begin{array}{c}\text { Total Pair } \\
\text { Reads }\end{array}$ & Mapped Pair Reads (Ratio) & Known Gene Num & Novel Gene Num \\
\hline A2 & 55729080 & $49972699(89.67 \%)$ & 26128 & 386 \\
A4 & 65402194 & $58874465(90.02 \%)$ & 26463 & 386 \\
N2 & 58025908 & $51001759(87.89 \%)$ & 26559 & 381 \\
N4 & 56371344 & $50670138(89.89 \%)$ & 25914 & 372 \\
\hline
\end{tabular}

1 
Table 2 (on next page)

List of PAL in the significant DEGs.

List of PAL in the significant DEGs. 


\begin{tabular}{|c|c|c|c|}
\hline Gene ID & Gene annotation & Fold change & FDR \\
\hline CmoCh03G012270 & $\begin{array}{l}\text { PREDICTED: phenylalanine } \\
\text { ammonia-lyase-like [Cucumis melo] }\end{array}$ & 0.439562 & $3.20 \mathrm{E}-05$ \\
\hline CmoCh03G012290 & $\begin{array}{l}\text { PREDICTED: phenylalanine } \\
\text { ammonia-lyase-like [Cucumis melo] }\end{array}$ & 0.343162 & $1.13 \mathrm{E}-28$ \\
\hline CmoCh07G009470 & $\begin{array}{c}\text { phenylalanine ammonia-lyase } 3 \\
\text { [Luffa aegyptiaca] }\end{array}$ & 0.458279 & 4.76E-08 \\
\hline CmoCh07G009540 & $\begin{array}{c}\text { phenylalanine ammonia-lyase } 3 \\
{[\text { Luffa aegyptiaca }]}\end{array}$ & 0.077247 & $1.86 \mathrm{E}-26$ \\
\hline CmoCh07G009550 & $\begin{array}{c}\text { phenylalanine ammonia-lyase } 3 \\
\text { [Luffa aegyptiaca }]\end{array}$ & 0.209537 & $1.47 \mathrm{E}-13$ \\
\hline CmoCh07G009560 & $\begin{array}{l}\text { PREDICTED: phenylalanine } \\
\text { ammonia-lyase-like [Cucumis melo] }\end{array}$ & 0.368197 & 4.93E-05 \\
\hline CmoCh20G005320 & $\begin{array}{c}\text { phenylalanine ammonia-lyase } 4 \\
{[\text { Luffa aegyptiaca }]}\end{array}$ & 2.000495 & 0 \\
\hline
\end{tabular}

1 


\section{Figure 1}

The aborted and normal pistils and differentially expressed genes (DEGs) that were significantly upregulated and downregulated between the aborted and normal pistils of pumpkin.

The aborted and normal pistils and differentially expressed genes (DEGs) that were significantly upregulated and downregulated between the aborted and normal pistils of pumpkin. (A) The aborted and normal pistils (bars $=1 \mathrm{~cm}$ ). (B) The number of upregulated and downregulated DEGs. (C) Volcano map for the gene expression. The $\mid \log 2$ (fold change) $\mid>1$ and FDR $<0.05$ were used as the cut-offs for significance.
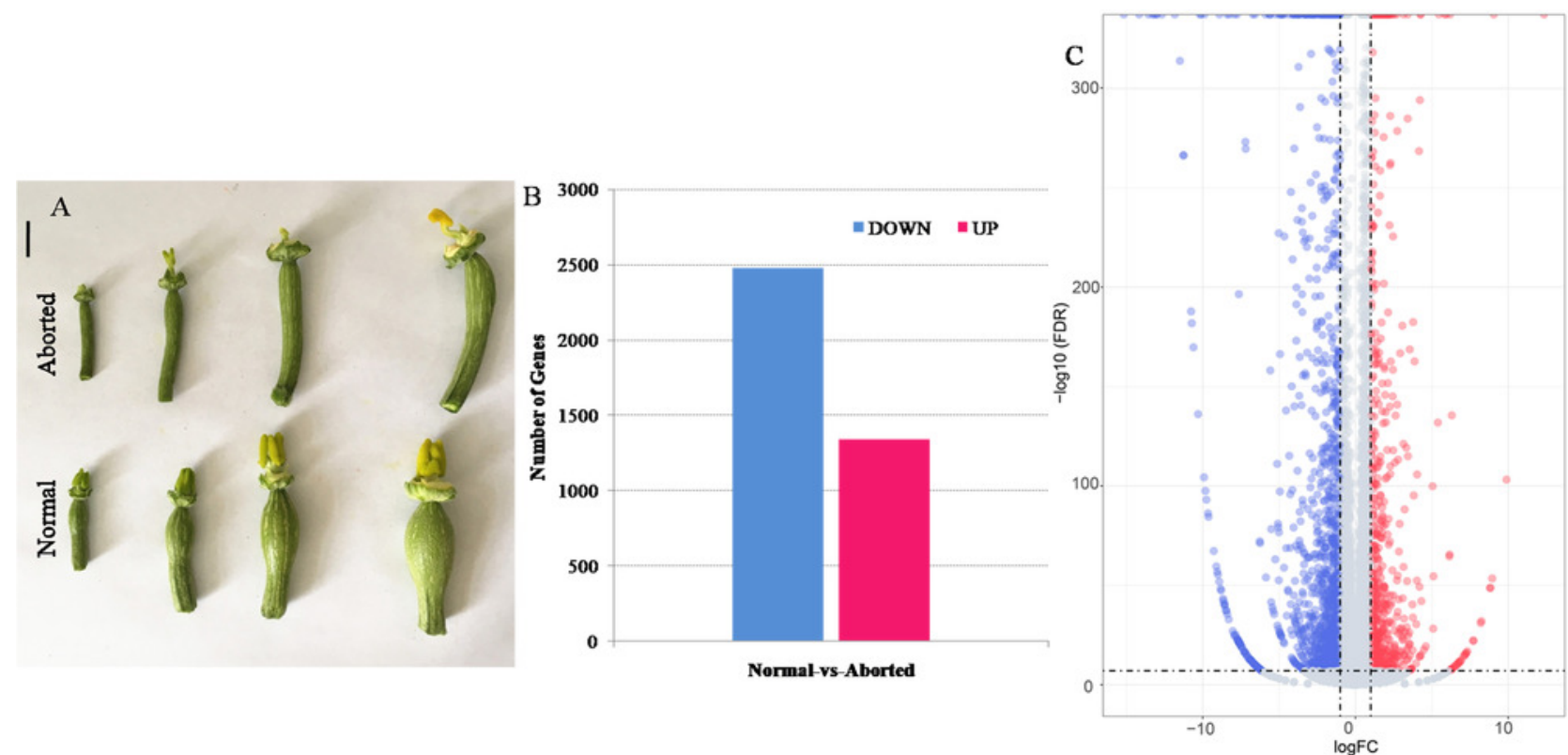
Figure 2

Gene ontology (GO) categories of differentially expressed genes (DEGs) between the aborted and normal pistils.

Gene ontology (GO) categories of differentially expressed genes (DEGs) between the aborted and normal pistils.

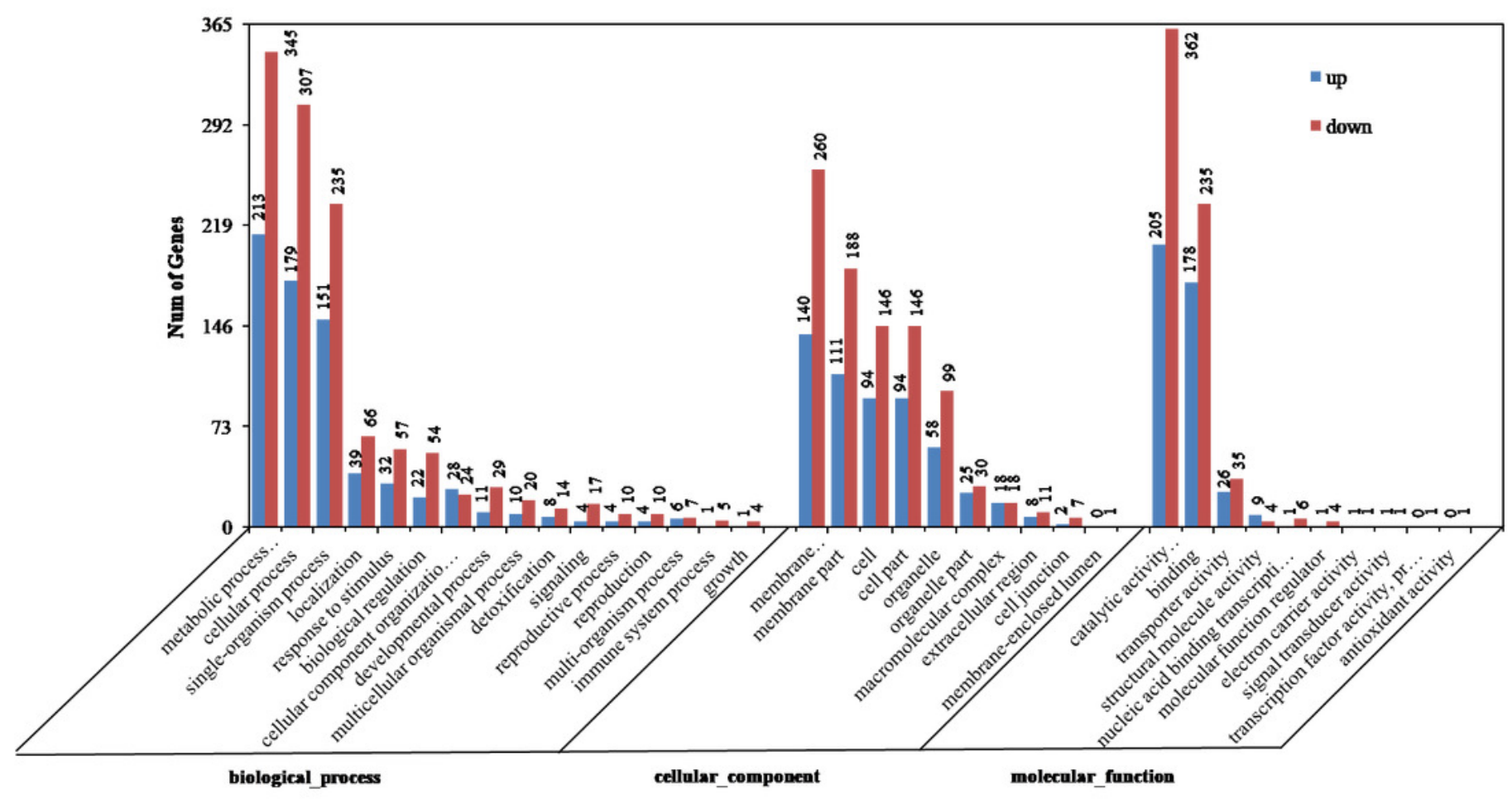




\section{Figure 3}

Kyoto encyclopedia of genes and genomes (KEGG) pathway enrichment of differentially expressed genes (DEGs) between the aborted and normal pistils.

\section{Kyoto encyclopedia of genes and genomes (KEGG) pathway enrichment of} differentially expressed genes (DEGs) between the aborted and normal pistils. Ggplot2 package for $\mathrm{R}$ language was used to generate this figure. The bigger the bubble, the more the DEGs. The smaller the pvalue, the more significant the KEGG enrichment. Rich Factor represents the ratio of DEGs number in the pathway to the total number of genes in the pathway. The larger the RichFactor, the higher the enrichment. 


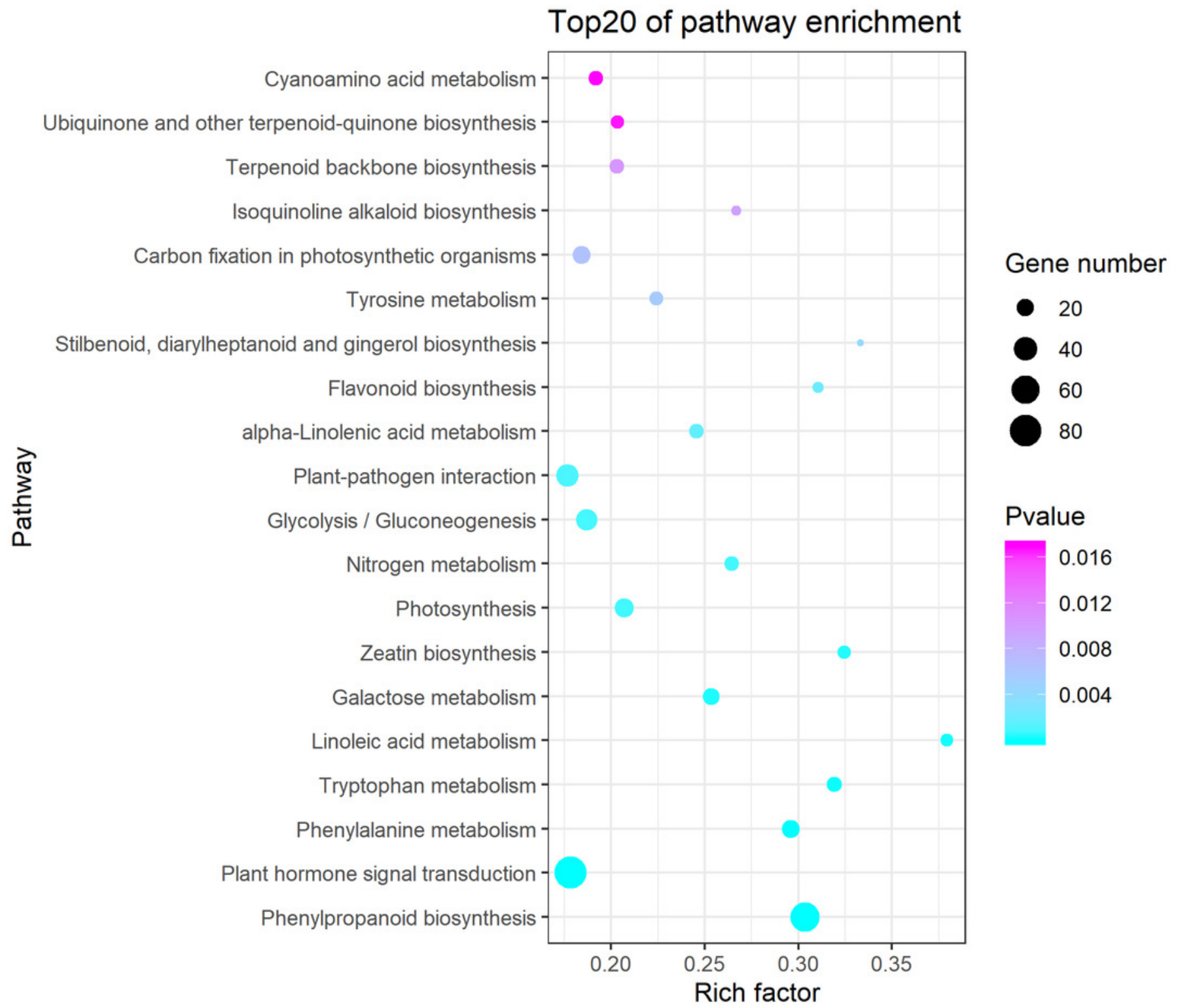




\section{Figure 4}

Verification of RNA-Seq Data by quantitative real-time PCR.

Verification of RNA-Seq Data by quantitative real-time PCR. (A) Relative normalized expression of 14 selected genes in the normal and aborted pistils using qRT-PCR. Error bars indicate the standard errors. AUX-IAA (auxin-responsive protein IAA), ARF (auxin response factor), TF (PIF4, phytochrome-interacting factor 4), ACO ( 1-aminocyclopropane-1carboxylate oxidation), ETR (ethylene receptor gene), ERDBF3 (ethyleneresponse DNA binding factor 3), ERTF10 (ethylene responsive transcription factor 10), AP2 (like ethyleneresponsive transcription factor), $\mathrm{TCH} 4$ (xyloglucan endotransglucosylase/hydrolase), CYCD3 (cyclin D3), JAR1 (jasmonic acid-amino synthetase), NPR1 (BTB/POZ domain and ankyrin repeat-containing protein NOOT2), TGA (TGACG-sequence-specific DNA-binding protein), PAL (phenylalanine ammonia-lyase). (B) Comparison of the expression ratios of 14 selected genes in using RNA-seq and qRT-PCR. 

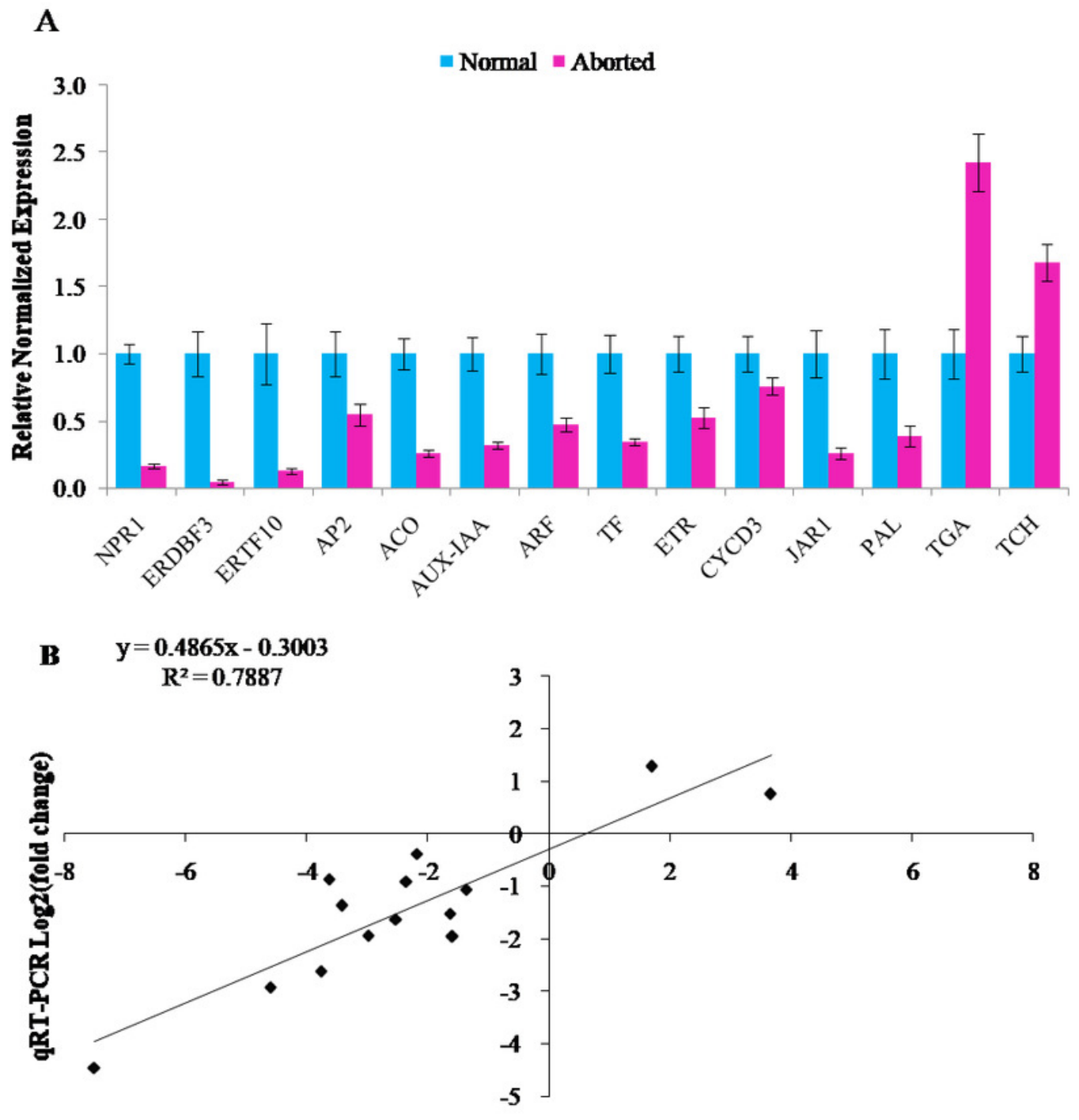

RNASSeq Log2(fold change) 


\section{Figure 5}

Expression levels of ethylene signal transduction candidate genes (ERDBF3, ERTF10, $A P 2$, and $E T R$ ) in different flower structures.

\section{Expression levels of ethylene signal transduction candidate genes (ERDBF3,} ERTF10, AP2, and ETR) in different flower structures. Error bars indicate the standard errors. ERDBF3 (ethyleneresponse DNA binding factor 3), ERTF10 (ethylene responsive transcription factor 10), AP2 (like ethylene-responsive transcription factor), ETR (ethylene receptor gene).

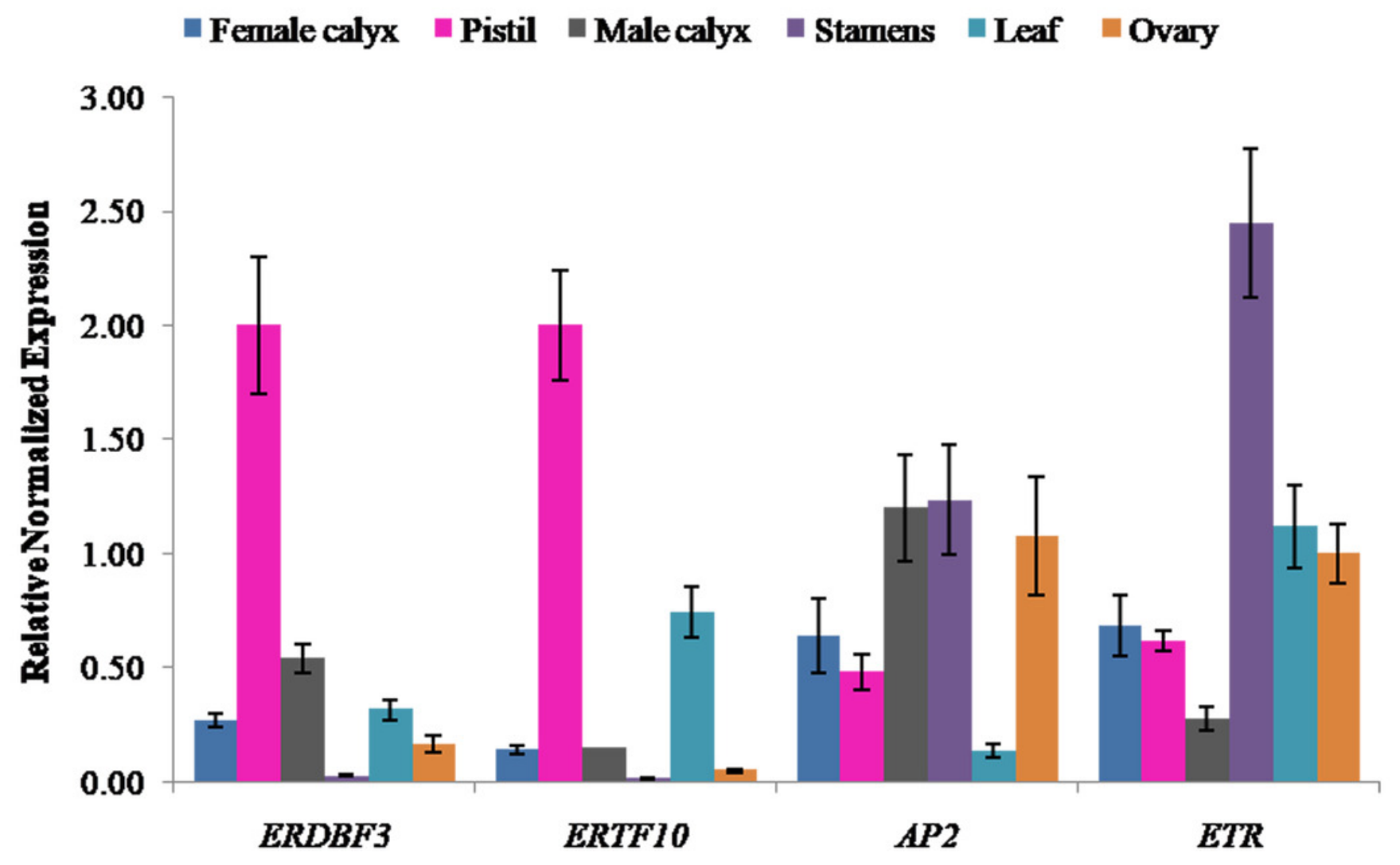

